\title{
Where is the Light? Tracing the Evolution of Bulges and Disks since $\mathbf{z} \sim 0.8 \dagger$
}

\author{
Lidia A. M. Tasca ${ }^{1}$, Laurence Tresse ${ }^{1}$, \\ COSMOS \& zCOSMOS Collaboration \\ ${ }^{1}$ Laboratoire d'Astrophysique de Marseille, CNRS-Université d'Aix-Marseille, 38 rue Frederic \\ Joliot Curie, 13388 Marseille Cedex 13, France \\ email: lidia.tasca@oamp.fr
}

\begin{abstract}
The chronology of galactic bulge and disk formation is studied by analysing the relative contributions of these components to the $B$ band rest-frame luminosity density (LD) at two different cosmological epochs. The luminosity function (LF) of the bulge and disk components at $\mathrm{z} \sim 0.8$ is computed on a galaxy subsample of the final zCOSMOS "bright" catalogue of roughly 20,000 objects with spectroscopic redshift in the COSMOS field. The comparison is then performed on galaxies in the local universe. Our preliminary results show that the LD in the disk component strongly decreases from $\sim 80 \%$ at $\mathrm{z} \sim 0.8$ to $\sim 50 \%$ at $\mathrm{z}=0$, the bulges having a specular behaviour. The observational constraints provided in this work are aimed to discriminate among competing scenarios of galaxy formation and evolution. An appropriate comparison with hydrodynamical semianalytical models will be considered in a future study to understand further the formation and evolution of galaxies.
\end{abstract}

Keywords. galaxies: evolution, galaxies: luminosity function, galaxies:bulges

\section{Overview}

This work aims to study the evolution since $\mathrm{z} \sim 0.8$ of the LD in the galactic bulge and disk components, to explore the chronology of their formation.

The VLT/VIMOS (Le Fèvre et al. 2003) observations of the brighter, magnitudelimited $\left(I_{A B}<22.5\right)$ part of the zCOSMOS redshift survey (Lilly et al. 2007), have been currently completed. The whole $1.7 \mathrm{deg}^{2}$ COSMOS field (Scoville et al. 2007) is covered. The final sample of 20, 707 objects, the so-called "20k bright", has been assembled providing the needed statistics. In addition, the high spectroscopic sampling rate $(\sim 70 \%)$ and the complete coverage of the COSMOS field with the Hubble Space Telescope (HST) Advanced Camera for Surveys (ACS) (Koekemoer et al. 2007), allows to perform on one side an accurate reconstruction of the density field (Kovač et al. 2010) and on the other a robust morphological classification (Tasca et al. 2009) and bulge/disk decomposition.

For this study we use a representative sample of 3266 galaxies, of which 2664 have environmental information, in the redshift range $0.7<\mathrm{z}<0.9$ to work on the $B$ band rest-frame, thus avoiding to apply a still unknown $\mathrm{K}$-correction for the bulge and disk components. Our data allows to quantify at $\mathrm{z} \sim 0.8$ : (i) the contribution of galactic bulges and disks to the total LD; (ii) the relative contribution of galaxies in underdense and overdense environments; (iii) the contribution of galactic bulges and disks to the total LD

$\dagger$ Based on observations obtained at the European Southern Observatory (ESO) Very Large Telescope (VLT), Paranal, Chile, as part of the Large Program 175.A-0839 (the zCOSMOS Spectroscopic Redshift Survey) 

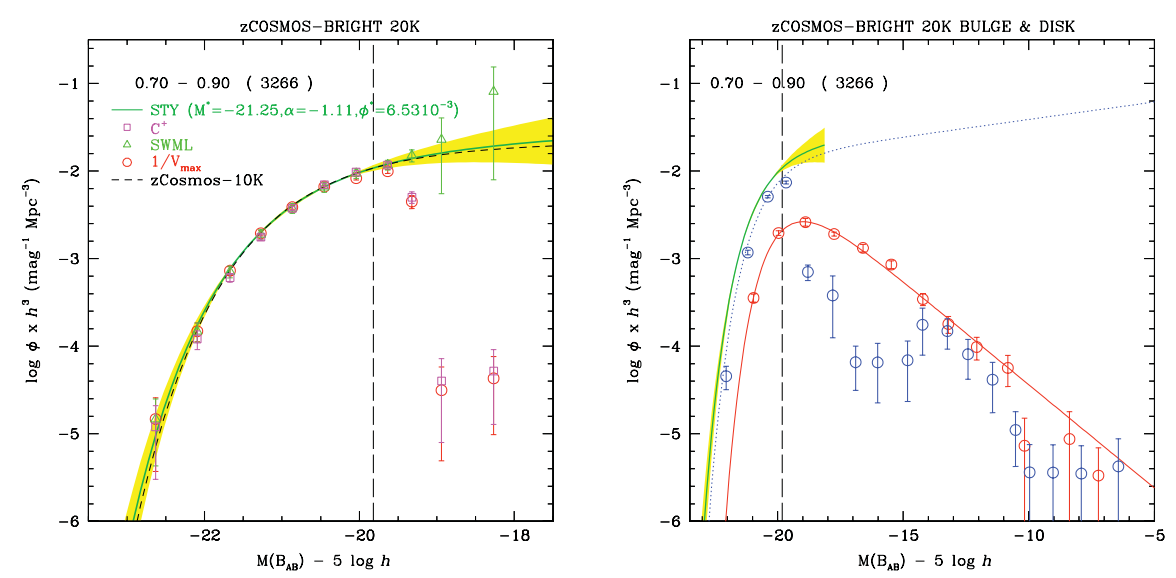

Figure 1. Left panel: global luminosity function in the $B$ rest-frame band at $0.7<\mathrm{z}<0.9$. The number of galaxies used is indicated in the label. The vertical dashed line represents the faint absolute limit considered in the $S T Y$ estimate. The luminosity function is estimated with different methods. The $S T Y$ results for this work over the 20k sample (solid line) and for the 10k sample (dashed line) (Zucca et al. 2009) are shown, as well as our results from the the $C^{+}$(squares), SWML (triangles) and $1 / V_{\max }$ (circles). The shaded region represents the $68 \%$ uncertainties of the parameters $\alpha$ and $M^{*}$. Right panel: bulge and disk luminosity function at $0.7<\mathrm{z}<0.9$ in the $B$ rest-frame band. The vertical dashed line represents the faint absolute limit considered in the $S T Y$ estimate for the global luminosity function. $1 / V_{\max }$ results for bulges (dots) and disks (circles) as well as $S T Y$ fits (solid line) of the global and bulge luminosity functions are shown. The disk luminosity function (dotted line) is obtained as difference between the global and the bulge ones.

in underdense and overdense environments. Throughout this paper we use the AB flux normalization and we adopt the set $\left(\Omega_{M}, \Omega_{\Lambda}, \mathrm{h}\right)=(0.3,0.7,0.7)$ for the cosmological parameters.

\section{Results}

Various studies have already computed the LD in bulges and disks in the local universe (e.g., Schechter 1986; Tasca \& White 2005a; Tasca \& White 2005b; Benson et al. 2006; Driver et al. 2007; Gadotti 2009; Tasca et al. submitted) using different samples, selections and methods. There is a general consensus that more than $50 \%$ of light in the local universe is contributed by stars in disks.

This study is, to our knowledge, the first attempt to compute the LD in bulges and disks at $\mathrm{z} \sim 0.8$. The LFs are computed using the "Algorithm for Luminosity Function" (ALF, Ilbert et al. 2005). Ilbert et al. (2004) demonstrated that the estimate of the global LF can be biased when the band used to compute the LF strongly differs from the rest-frame band in which the galaxies are selected. This effect is less important when computing the LF of galaxies divided by type because the effect of $\mathrm{K}$-correction is minimized. When considering galaxy bulges and disks this bias is also expected to be less important than for the whole population, nevertheless we prefer to restrict our analysis to the interval $0.7<\mathrm{z}<0.9$ corresponding to the $B$ band rest-frame, because the $\mathrm{K}$-corrections are small.

The global $B$ band LF at $0.7<\mathrm{z}<0.9$ is shown in Fig. 1 (left panel). The corresponding LFs for the bulge and disk components (Fig. 1, right panel) show that, while the LF of 

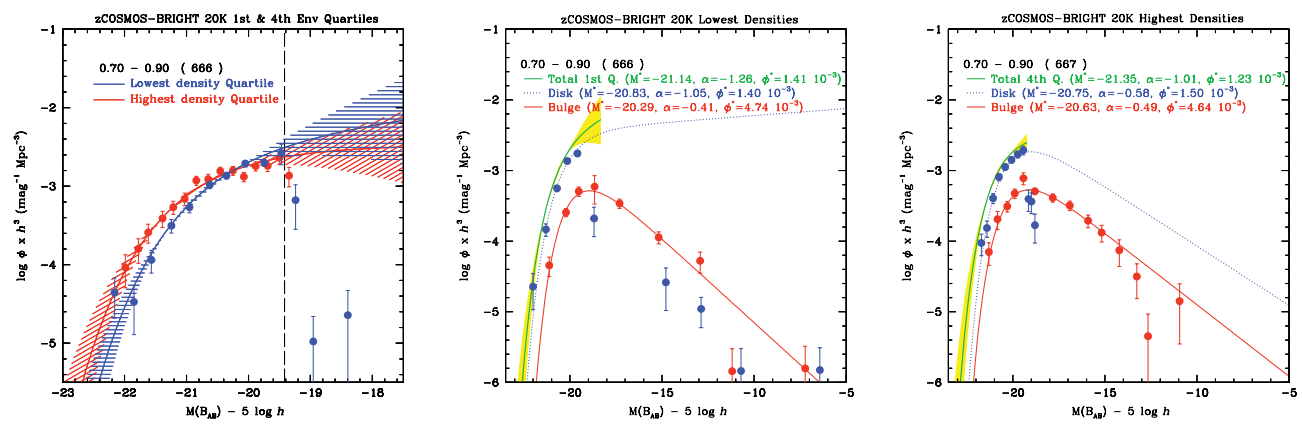

Figure 2. Left panel: global luminosity function in the lowest (blue) and highest (red) quartile of the density distribution in the redshift interval $0.7<\mathrm{z}<0.9$. Overdensities are derived from the 5 th nearest neighbours. The vertical dashed line represents the faint absolute limit considered in the $S T Y$ estimate. Middle and right columns: luminosity functions in the lowest and highest quartile of the density distribution. In each panel the global luminosity function is shown in black, while the luminosity function for the bulge and disk components are drawn in red and blue respectively. The meaning of symbols and lines is the same as in Fig. 1.

the bulge component follows a Schechter function (Schechter 1976) with a sharp decrease of the density at faint magnitudes, the LF of the disk component is clearly incomplete below the magnitude limit of the survey. Since the capability to measure the flux in disks is strongly limited by the low surface brightness, this incompleteness is large. The good knowledge of the global LF allows to retrieve (by difference with the bulge LF) the LF of disks at faint magnitudes, which shows a slope of -1.1 . The LD is then obtained by integrating the LFs. Stars in the galactic disk components decrease their contribution to the global LD of $\sim 30 \%$ since $\mathrm{z} \sim 0.8$. The study of the physical mechanisms responsible of the observed evolution is undergoing and allow to discriminate among competing scenarios of galaxy formation and evolution.

Using the zCOSMOS 10k catalogue we have already shown (e.g., Tasca et al. 2009; Bolzonella et al. 2010; Pozzetti et al. 2010; Peng et al. 2010, among others) that stellar mass is the main driver of the observed dependencies of galaxy properties on the environment. Still the environment playing an important, although secondary, role.

To better understand the role of the environment, we derive the LF for galaxies in the interval $0.7<\mathrm{z}<0.9$ in underdense and overdense regions, using as density estimator the 5 th nearest neighbour as computed in Kovač et al. (2010). The galaxy density distribution is divided into equipopulated quartiles and we consider galaxies in the two extreme tails in order to accentuate the differences between the two environments. Zucca et al. (2009), studying the evolution of the LF to $\mathrm{z} \sim 1$ for the zCOSMOS 10k sample, found that the LF of galaxies in overdense regions always has a brighter $M^{*}$ and a flatter slope. This is confirmed by our analysis (Fig. 2, left panel) which, having the advantage of higher statistics (i.e., we are using the $20 \mathrm{k}$ catalogue instead than the $10 \mathrm{k}$ ), allows to further reduce the redshift range explored (smaller redshift bin).

Does the environment also play a role on the formation and evolution of galactic bulges and disks? We explore in Fig. 2 the LF of bulges and disks at $\mathrm{z} \sim 0.8$ in low (middle panel) and high (right panel) density environments. The bulge emissivity is estimated at $18 \pm 1 \%$ independent on the environment. 


\section{Discussion \& Conclusions}

The evolution of the fraction of light in the bulge and disk components since $\mathrm{z} \sim$ 0.8 is computed by comparison with similar estimates in the local universe. From our representative sample of $\sim 3200$ galaxies, we estimate a strong decrease, from $\sim 80 \%$ to $\sim 50 \%$, of the fraction of light coming from the disk components of galaxies in the latest $7 \times 10^{9}$ yrs. A strong and opposite evolution for bulges and disks is detected. A preliminary estimate of the bulge emissivity in the highest and lowest density quartile of the density distribution, of $\sim 18 \%$ independent of the environment, indicates that the environment does not play an "easy to identify" role.

Does the $B$-band emissivity gradually shift from disks to bulges towards low-z? The $B$-band emissivity is tightly related to $\mathrm{H} \alpha$, i.e. SFR (Tresse et al. 2002) thus the galaxy population is increasing its SFR density within bulge, either via star formation and/or number density increase. The sharp decrease of the recent history of the SFR (e.g. Tresse et al. 2007) is mainly linked to the star formation activity within disks, the rest $-B$ band light being less dominated by starbursts but more by long-lived stars.

With the aim to finalise this preliminary work, we are repeating the analysis (Tasca et al. in preparation) distinguishing between pure bulge systems (elliptical galaxies) and spheroidal components (bulges of spirals) to investigate whether the environment differently acts on them and because earlier Hubble types are known to exhibit more nuclear SF than late types (Kennicutt 1998). Finally, an accurate and meaningful comparison with cosmological hydrodynamical simulations is needed to discriminate among different scenarios of galaxy formation and evolution.

\section{Acknowledgements}

LT acknowledge support from the University of Provence and thank IAU LOC and SOC for this stimulating conference in such an extraordinary country. We thank CNES and PNC for support to the COSMOS project. This work benefited from support from the French National Computing Centre (CINES), for providing part of the necessary computational resources. This research has made use of data from zCOSMOS \& HSTCOSMOS projects, held in the HST-COSMOS \& zCOSMOS databases operated by Cesam, Laboratory of Astrophysics of Marseille.

\section{References}

Benson, A. J., et al. 2006, MNRAS, 379, 841

Bolzonella, M., et al. 2010, A\&A, 524, A76

Driver, S. P., et al. 2007, ApJ, 657, 85

Gadotti, D. A. 2009, MNRAS, 393, 1531

Kennicutt, R. C., Jr. 1998, ARA\&A, 36, 189

Koekemoer, A. M., et al. 2007, ApJS, 172, 196

Kovač, K., et al. 2010, ApJ, 708, 505

Ilbert, O., et al. 2004, MNRAS 351, 541

Ilbert, O., et al. 2005, A\& $A, 439,863$

Le Fèvre, O., Saisse, M., \& Mancini, D., et al. 2003, Proc. SPIE, 4841, 1670

Lilly, S. J., Le Fèvre, O., \& Renzini, A., et al. 2007, ApJS, 172, 70

Pozzetti, L., et al. 2010, A\&AA, 523, 13

Peng, Y.-j., et al. 2010, ApJ, 721, 193

Schechter, P. 1976, ApJ, 203, 297

Schechter, P. L. 1986, AJ, 94, 563

Scoville, N. Z., et al. 2007, ApJS, 172, 1 
Tasca, L. A. M., \& White, S. D. M. 2005, Multiwavelength Mapping of Galaxy Formation and Evolution, 465

Tasca, L. A. M., \& White S. D. M. 2005, arXiv:astro-ph/0507249v1

Tasca, L. A. M., et al. 2009, A\&BA, 503, 379

Tasca, L. A. M., et al., submitted

Tasca, L. A. M. et al., in preparation

Tresse, L., et al., 2007, A\&A, 472, 403

Tresse, L., Maddox, S. J., Le Fèvre, O., \& Cuby, J.-G. 2002, MNRAS, 337, 369

Zucca, E. et al., 2009, A\&A A, 508, 1217 